\title{
News-Topic Oriented Hashtag Recommendation in Twitter Based on Characteristic Co-occurrence Word Detection
}

\author{
Feng Xiao, Tomoya Noro, and Takehiro Tokuda \\ Department of Computer Science, Tokyo Institute of Technology, Japan \\ Meguro, Tokyo 152-8552, Japan \\ \{xiao, noro, tokuda\} att.cs.titech.ac.jp
}

\begin{abstract}
Hashtags, which started to be widely used since 2007, are always utilized to mark keywords in tweets to categorize messages and form conversation for topics in Twitter. However, it is hard for users to use hashtags for sharing their opinions/interests/comments for their interesting topics. In this paper, we present a new approach for recommending news-topic oriented hashtags to help Twitter users easily join the conversation about news topics in Twitter. We first detect topic-specific informative words co-occurring with a given target word, which we call characteristic co-occurrence words, from news articles to form a vector for representing the news topic. Then by creating a hashtag vector based on tweets with the same hashtag, we calculate the similarity between these two vectors and recommend hashtags of high similarity scores with the news topic. Experimental results show that our approach could recommend hashtags which are highly relevant to the news topics, helping users share their tweets with others in Twitter.
\end{abstract}

Keywords: Social Media, hashtags, tweet, characteristic co-occurrence word, clustering, news topic, Twitter.

\section{Introduction}

News articles, as a traditional medium for distributing information all over the world, have been increasingly impacted by a new way of information delivery called social media. Social networking services, such as Twitter, Facebook, and Digg, provide plenty of ways for users to share information with others. For example, since Twitter was launched in July 2006, the number of users and messages, called tweets, increased dramatically. Most of tweets in Twitter often concern topics of headline news or persistent news [1], making Twitter a suitable and important data resource for posting/receiving breaking news and opinions.

Currently, a lot of tools/functions are provided to help news agencies/users easily share news information in Twitter. Most of news websites provide Tweet Button in their Web pages to help readers easily share news articles with their followers. Retweet function greatly accelerates the spreading speed of information and mention function using @username help Twitter users exchange information directly with 
others. Hashtags (the \# symbol prefixed to a short character string) are widely used to categorize and joint tweets together based on a certain topic and make your tweets more easily searchable by other users with the same interest.

However, it is hard for Twitter users to use hashtags in their tweets properly when they want to share contents or their opinions/interests/comments for news topics. For many news websites, they do not provide any hashtag in tweets when users click the Tweet Button on their Web pages, which means users' sharing could only be seen by their followers and might not reach far to the others. Other news websites append hashtags automatically while most of them are not for the purpose of helping users share their tweets or too unique. Some of news websites use their formal name (such as "\#CNN") as the hashtag in every tweet from the Tweet Button of their news Web page no matter what the topic the news article reports. Such a kind of hashtag could only help these sites watch the information spreading in Twitter or promote reputation for advertising. Other news websites like Yahoo! Japan News provide hashtags such as "\#yjfc_wall_street_protest" when users post tweets from news Web pages reporting protest in Wall Street while such a kind of hashtags might only be used by Yahoo! Japan readers and is not widely used by other users.

It is also very hard for Twitter users to create/select proper hashtags by themselves. Users try to create hashtags which they took for granted that these hashtags should be widely used for topics while the truth might be just on the contrary. For example, "\# Wall_Street_Protest" might be thought as a meaningful hashtag used in tweets talking about the protest in Wall Street, but we found that no one uses this hashtag in his tweets up to the point of writing this paper. Users could search for some topic-related keywords and read all those responded tweets to find hashtags that relate to the topic. However, there might be too many hashtags contained in those responded tweets, relating to more than one topic, that users may have no idea which hashtags should be chosen. If all else fails, users may have to add the \# symbol prefixed to every word in their tweets; wishing one of these hashtags could be the one which is widely used by others for the topic in Twitter. However, such a behavior would make the tweets hard to read and impolite. The users may be taken as tweet spammers.

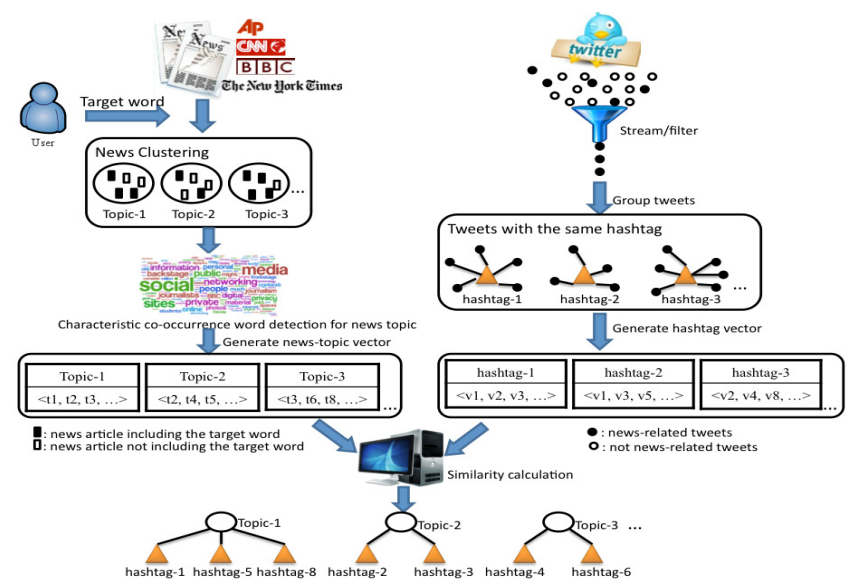

Fig. 1. System structure of the news-topic oriented hashtag recommendation 
In this paper, we present a new approach for recommending hashtags to the user who wants to join the conversation for a news topic by using hashtags in Twitter after he/she searches for the news topic by a keyword (we refer to it as target word in this paper) but has no idea which hashtag could be used. The whole system structure is depicted in Figure 1. In our approach, we first collect news articles and news-related tweets published in a certain period of time concurrently. Then news articles are clustered into topics. News-related tweets including mentioned or tagged screen names of news agencies (e.g. @CNN, \#CNN) are concatenated based on the hashtags and a vector for representing each hashtag is created. After the target word has been provided, news topics that relate to the target word are selected and a vector is created for representing each of the news topics. We calculate the similarity score between each news-topic vector and each hashtag vector, and hashtags with high similarity scores are recommended for the news topic.

To represent news topics that relate to the target word, we propose a new method named Probabilistic Inside-Outside Log (P-IOLog) method to detect characteristic co-occurrence words from news articles. Characteristic co-occurrence words are topic-specific words which provide information for news topics related to the target word and could be detected based on the assumption that characteristic co-occurrence words should often co-occur with the target word in news articles while they are less likely to appear when the target word is not contained. Words with their scores detected by our P-IOLog method are used to create news-topic vectors. We also extend this P-IOLog method for hashtags to detect informative words co-occurring with the hashtags in tweets and all these detected words with their scores are used to create hashtag vectors. We refer to the extended method as P-IOLogH method.

Notice that our approach is trying to recommend hashtags which have been created and used in tweets. New hashtag generation is not our goal. Also we are trying to help users who want to share their opinions/interests/comments and join conversations for news topics in Twitter. Other kinds of Twitter users, such as bots, are not considered.

The organization of the rest of this paper is as follows. We present related work in the next section. In Section 3, we describe our method for detecting characteristic co-occurrence words with the target word, named Probabilistic Inside-Outside Log (P-IOLog) method, and how to create news-topic vectors based on these detected words. In Section 4, we propose two methods (TF-IHF and P-IOLogH) to weight words co-occurring with hashtags for creating hashtag vectors and explain how to get recommended hashtags for news topics. Experimental results and evaluations are described in Section 5. In Section 6 we make the conclusion with directions for future research.

\section{Related Work}

Hashtags in Twitter are one special type of a more general concept, tag, which is an important feature for many social networking services. People could create tags with few taxonomic constraints to categorize resources for later browsing, or to describe resources for searching. Many approaches for tag recommendation in social networking services have been proposed recently. They are mainly classified into two classes. 
One class of these approaches focuses on the relationship between tags and their associated resources, and recommends tags to a newly added resource. One application in this class is the tag recommendation system for weblog. Brooks et al. [2] try to select words in blog posts that have high TF-IDF scores to be used as tags, and find that those tags are more representative than human-assigned ones. Mishne G. [3] and TagAssist [4] recommend tags to a new blog post by providing tags in those old blog posts which have high TF-IDF similarity with the new one. These approaches recommend tags in similar resources by using techniques from Information Retrieval (e.g. TF-IDF). However, these methods are no longer effective for recommending hashtags in Twitter because TF-IDF reduces the chance of relevant tweets to be selected since the tweet length is limited and their contents have less information [5].

Other approaches exploit tag co-occurrence patterns through a history of tag assignments in a collaborative tagging environment when the resource with which the tag was associated is hard to retrieve such as audio, video, and image. Sigurbjornsson et al. [6] recommend tags for each user-defined tag for photos based on tag cooccurrence in Flickr. Wartena et al. [7] proposed another approach to calculate the similarity between tag co-occurrence distribution and the user profile. Tags with high similarity are recommended to the user. Belem et al. [8] extended tag co-occurrence exploiting and considered about terms extracted from other textual features such as title and description. All these approaches are based on two assumptions: tags are assigned to resources beforehand and most of resources have two or more tags ${ }^{1}$. However, most of tweets in Twitter only contain one or even no hashtag. For example, in all news-related tweets collected on December $20^{\text {th }} 2011,88.6 \%$ of tweets contain one or no hashtag. Exploiting tag co-occurrence in tweets becomes impossible due to the small number of tweets containing two or more hashtags.

Recently, researchers found out that hashtags in Twitter play a different role compared to tags in other social networking services. Huang et al. [9] compared user tagging behaviors between Twitter and Delicious. They found out that hashtags in Twitter are used to join discussions on existing topics while in Delicious tags are used to re-access resources. Our approach is based on the conversational nature of hashtags and tries to recommend hashtags to help users join the conversation about the news topic so that users do not need to be "exposed" to too many hashtags

Approaches for hashtag retrieval/recommendation in Twitter have been proposed while there are some problems still existed. Weng et al. [10] proposed methods for modeling the interestingness of hashtags by studying how hashtags are discussed within and across communities, but they do not correlate hashtags with topics in which users are interested. Correa et al. [11] proposed a new approach for recommending tags to other social networking services such as Flickr and YouTube, using hashtags and terms in tweets. Our approach is different because we correlate Twitter with traditional news media, not other social networking services. Efron M. [12] and Wagner C. et al. [13] proposed new approaches to retrieve useful hashtags after a keyword is given. However, one keyword may relate to more than one topic and all

\footnotetext{
${ }^{1}$ Flickr allows its users to add 75 tags per photo at most; In YouTube the total length of your tag list is limited to 500-character.
} 
hashtags related to different topics would be mixed together. Also, ranking hashtags based on their in-degree in [13] would make some general hashtags (e.g. \#tech) be ranked higher, which is still not helpful. Zangerle et al. [14] proposed a method to recommend hashtags to users' inputted contents by calculating similarities between newly inputted contents and old tweets based on TF-IDF. Hashtags which frequently appear in old tweets with high similarities are recommended. However, similarity among tweets is hard to decide by simply relying on those common words since semantics and synonyms are not considered. Also, due to the huge number of tweets, TFIDF is no longer a good choice because the IDF part would dominate the final score, assigning large score to the word which appears scarcely (e.g. misspelling).

Other approaches interweaving traditional news media with social networking services have also been proposed for Topic Detection and Tracking $[15,16]$, news recommendation [17], and user profile construction [18]. To the best of our knowledge, our approach is the first one trying to recommend hashtags for news topics in which users are interested.

\section{Characteristic Co-occurrence Word for News Topic}

A news topic is a group of news articles published in a period of time (for example: one day) reporting about the same recent event in the world. Traditional method for representing the news topic is to define a centroid vector which is calculated by averaging vectors of all news articles in this topic under the Vector Space Model [19]. Each vector dimension corresponds to a separate term in news articles and term weights are calculated by the TF-IDF. Although TF-IDF works well in many tasks such as Information Retrieval, it is no longer the best choice for our approach. Firstly, TF-IDF is a query-independent term weighting method, which means the term weight doesn't change no matter what the query is. Secondly, TF-IDF is a topic-independent method. The term which appears in most news articles of a news topic should be weighted higher while TF-IDF could not reflect this idea. At last, even news articles of the same news topic may share many common terms, a news topic may contain thousands of separate terms, which would greatly increase the computation.In order to solve these problems, we propose a new method for detecting characteristic cooccurrence words of news topics with the target word which is queried by users. Characteristic co-occurrence words are those words which provide important information for news topics related to the target word. Our method for detecting characteristic cooccurrence words is based on two assumptions:

- Characteristic co-occurrence word $w$ should often co-occur with the target word $t$ in news articles; we take it as the Inside part.

- Characteristic co-occurrence word $w$ should not always appear in news articles without the target word $t$; we take it as the Outside part.

Based on these two assumptions, words often appearing in news articles including the target word while being less likely to appear in news articles without the target word are taken as the characteristic co-occurrence words. However, all of news articles containing the target word do not always deal with the same news topic and characteristic 
co-occurrence words related to different news topics will be mixed together. Also, some words which often co-occur with the target word regardless of the news topic (e.g. Obama and White House) should be excluded since those words provide little information for a specific news topic.

In order to solve these problems, we firstly clustered news articles and those articles which related to the same news topic are hopefully put into the same group while news articles related to different news topics would be partitioned into different ones. Then we can detect characteristic co-occurrence words for every news topic related to the target word separately without a mixture of words. The procedure is depicted in the Figure 2(a). When dealing with one news topic, all news articles containing the target word in this topic are taken as the Inside part while all the other news articles regardless of existence of the target word are taken as the Outside part. Words co-occurring with the target word regardless of the topic are excluded under this way.

To reflect our idea, we introduce Probabilistic Inside-Outside Log method to calculate the score of word $w$ co-occurring with the target word $t$ in topic $c$ as follows.

$$
\begin{gathered}
\mathrm{P}-\mathrm{IOL} \log (w, t, c)=\log \frac{\left(1-s_{p}\right) P(w \mid t \wedge c)+s_{p}}{\left(1-s_{p}\right) P(w \mid \neg(t \wedge c))+s_{p}} \\
P(w \mid t \wedge c)=\frac{d f(w \wedge t \wedge c)}{d f(t \wedge c)} \\
P(w \mid \neg(t \wedge c))=\frac{d f(w \wedge \neg(t \wedge c))}{d f(\neg(t \wedge c))}=\frac{d f(w)-d f(w \wedge t \wedge c)}{N-d f(t \wedge c)}
\end{gathered}
$$

where $d f(w)$ is the number of news articles containing $w . d f(w \wedge t \wedge c)$ is the number of news articles not only containing both $w$ and $t$, but also belonging to the news topic $c$. $d f(\mathrm{w} \wedge \neg(t \wedge c))$ is the number of news articles containing $w$ but not containing $t$ or not being assigned to news topic $c$ (Figure 2(b)). $\mathrm{N}$ is the total number of news articles and $s_{p}$ is a smoothing parameter which ranges from 0 to 1 . The P-IOLog score will vary from $\log \left(s_{p}\right)$ to $\log \left(1 / s_{p}\right)$. The larger the score is, the more likely the word $w$ is a characteristic co-occurrence word with the target word $t$ for news topic $c$.

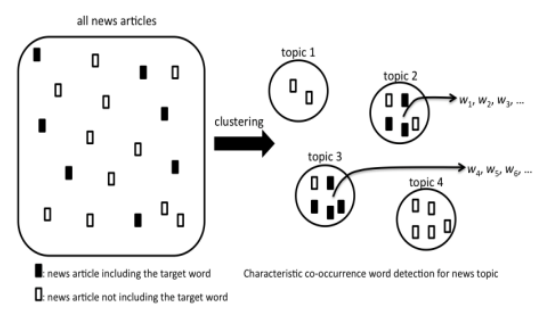

(a) Characteristic co-occurrence word detection for news topic method

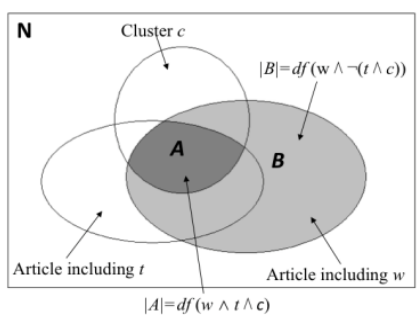

(b) Probabilistic Inside-Outside Log

Fig. 2. Procedure of characteristic co-occurrence word detection for news-topic 
For every news topic related to the target word, we select the target word $t$ and top- $(n-1)$ words whose P-IOLog scores are larger than the others to create a newstopic vector. The news-topic vector's dimension is $n$ and the term weight for every dimension is defined as the normalized P-IOLog score. They are created as follows.

$$
\begin{gathered}
\overrightarrow{n t}(t, c)=<c t_{1}, c t_{2}, \ldots, c t_{n}> \\
c t_{i}=\frac{\mathrm{P}-\mathrm{IOLog}\left(w_{i}, t, c\right)}{\sqrt{\left(\mathrm{P}-\operatorname{IOLOg}\left(w_{1}, t, c\right)\right)^{2}+\ldots+\left(\mathrm{P}-\mathrm{IOLOg}\left(w_{n}, t, c\right)\right)^{2}}}
\end{gathered}
$$

where $c t_{i}$ is the normalized weight for word $w_{i} \cdot \overrightarrow{n t}(t, c)$ is the vector for representing news topic $c$ that relates to the target word $t$. The weight of the target word $t$ is assigned as the maximum value of P-IOLog.

Compared with other methods for calculating co-occurrence coefficients, our PIOLog method is an asymmetric method while some others are symmetric measures (e.g. Jaccard). However, for detecting characteristic co-occurrence words which are topic-specific, detected words should be query and topic-dependent while symmetric measures could not reflect this idea. Also, unlike other asymmetric methods such as DF-IDF, our method considers not the raw word co-occurrence frequency, but the co-occurrence probability in news articles with/without the target word. Detailed experiments of comparison could be found in [20].

\section{News-Topic Oriented Hashtag Recommendation}

In order to find news-topic oriented hashtags, one intuitive way is to retrieve tweets related to a news topic and recommend commonly used hashtags among these tweets. However, tweet content is limited within 140 characters, which means there is far not enough information in a single tweet to decide whether the tweet relates to the news topic. Also, traditional way as TF-IDF for weighting terms is no longer effective for short text which has been pointed out [5].

Our method to recommend news-topic oriented hashtags is based on two assumptions:

- Tweets containing recommended hashtags should relate to the news topic.

- Recommended hashtags should be widely used by Twitter users when they discuss the news topic.

For the first assumption, when Twitter users are discussing a news topic, some informative words of this news topic would be likely to be used in their tweets. The second assumption means that when one hashtag is widely used for a news topic in Twitter, users would use this hashtag to exchange information about the news topic from different perspectives, which means more informative words of the news topic are likely to be used in users' tweets. 
Based on these two assumptions, we concatenate all tweets which contain the same hashtag and a hashtag vector is created based on concatenated contents of tweets. Each dimension of the hashtag vector corresponds to a separate term in the concatenated contents of tweets. We propose two different methods to calculate the term weight for the hashtag vector.

\subsection{Term Frequency-Inverted Hashtag Frequency (TF-IHF)}

Term Frequency-Inverted Hashtag Frequency (TF-IHF) is a variation of TF-IDF measure which considers not only the term frequency in a single document, but also the general importance of terms. TF-IHF score will be calculated as follows.

$$
\mathrm{TF}-\operatorname{IHF}\left(w_{i}, h t_{j}\right)=\mathrm{TF} \times \mathrm{IHF}=\frac{n_{i}}{\sum_{k} n_{k}} \times \log \frac{|\mathrm{HT}|}{\left|h_{d}: w_{i} \in h_{d}\right|}
$$

where $w_{i}$ is the term to which the TF-IHF score corresponds and $h t_{j}$ is the hashtag. $|\mathrm{HT}|$ gives the total number of hashtags in the dataset and $h_{d}$ means the concatenated contents of tweets containing the same hashtag $h t_{d}$ where $\mathrm{d} \in\{1, \ldots,|\mathrm{HT}|\} . n_{i}$ is the number of times $w_{i}$ appears in $h_{j}$ and $k$ is the total number of separate terms in $h_{j}$. TF-IHF value ranges from 0 to $\log |\mathrm{HT}|$ and high value would be reached when $w_{i}$ frequently appears in the concatenated contents of tweets for hashtag $h t_{j}$ and rarely cooccur with other hashtags.

However, TF-IHF does not consider about the number of tweets containing both term $w_{i}$ and hashtag $h t_{j}$, which might cause a bias towards terms appearing many times in a few tweets with the hashtag. These terms might get higher TF-IHF scores compared to the others which appear in more tweets with the hashtag but only occur once in each tweet.

\subsection{Probabilistic Inside-Outside Log Method for Hashtags (P-IOLogH)}

To conquer the problem in TF-IHF method, we extend our idea in section 3 and apply those two assumptions for tweets. Our Probabilistic Inside-Outside Log method for hashtags $(\mathrm{P}-\mathrm{IOLogH})$ takes those tweets containing hashtag $h t_{j}$ as the Inside part and tweets containing other hashtags as the Outside part. Terms which often co-occur with hashtag $h t_{j}$ in tweets of the Inside part while not so often appear in tweets with other hashtags in the Outside part would be taken as the characteristic co-occurrence words with the hashtag and assigned a high term weight. P-IOLogH score will be calculated as follows.

$$
\begin{gathered}
\mathrm{P}-\mathrm{IOLogH}\left(w_{\mathrm{i}}, h t_{j}\right)=\log \frac{\left(1-s_{p}\right) P\left(w_{i} \mid h t_{j}\right)+s_{p}}{\left(1-s_{p}\right) P\left(w_{i} \mid \neg h t_{j}\right)+s_{p}} \\
\mathrm{P}\left(w_{i} \mid h t_{j}\right)=\frac{\text { TweetFrequency }\left(w_{i} \wedge h t_{j}\right)}{\text { TweetFrequency }\left(h t_{j}\right)}
\end{gathered}
$$




$$
\mathrm{P}\left(w_{i} \mid \neg h t_{j}\right)=\frac{\text { TweetFrequency }\left(w_{i}\right)-\text { TweetFrequency }\left(w_{i} \wedge h t_{j}\right)}{N T_{h t}-\text { TweetFrequency }\left(h t_{j}\right)}
$$

where TweetFrequency $\left(w_{i} \wedge h t_{j}\right)$ gives the number of tweets containing both term $w_{i}$ and hashtag $h t_{j}$. $N T_{h t}$ means the total number of tweets containing hashtags in the database. The P-IOLogH score get a value ranging from $\log \left(s_{p}\right)$ to $\log \left(1 / s_{p}\right)$ with $s_{p}$ as the smoothing parameter. The larger the P-IOLogH score is, the more likely term $w_{i}$ is the characteristic co-occurrence word with the hashtag and be more informative.

\subsection{Hashtag Vector Creation and Similarity Calculation}

For every hashtag, tweets which contain the same hashtag are concatenated to form contents. Term weight for each separate term in the concatenated contents would be calculated by TF-IHF and P-IOLogH. Top- $n$ words whose term weights are larger than the others are selected to create the hashtag vector. Value in each dimension of the hashtag vector is normalized to make the norm of the vector equals to 1 .

Our two assumptions for hashtag recommendation result in a high cosine similarity between the news-topic vector and the hashtag vector. Hashtags whose vectors have high similarity with the news-topic vector should be recommended to users who wish to join the conversation about the news topic in Twitter.

\section{Experiment and Evaluation}

\subsection{Description of the Dataset}

In order to validate the effectiveness of our approach on real-world data, news dataset and news-related tweet dataset are prepared for the experiment. News dataset contains 10,855 news articles crawled from 96 news sites ( 21 countries/regions) on December $20^{\text {th }}, 2011$. However, due to the huge number of tweets for different topics in Twitter, it is hard to retrieve all those tweets related to news topics. Our solution is to manually select 54 active Twitter accounts of news agencies and collect tweets which are posted by these accounts or contain mentioned/tagged screen names of these accounts (e.g. @CNN, \#CNN) by using Twitter Streaming API [21]. At last we collected 124,481 tweets on December $20^{\text {th }}, 2011$ to create our news-related tweet dataset. Although there might be some other tweets related to news topics, collecting those tweets by formal Information Retrieval technologies is no longer effective due to the limited length of a tweet and a trade-off has to be made.

\subsection{Experimental Setup}

News articles in our news dataset are parsed by using TreeTagger [22] and Stanford Named Entity Recognizer (SNER) [23]. All nouns, proper nouns, foreign words, verbs and adjectives are picked up for representing each news article under Vector 
Space Model as TF-IDF vector. Then all news articles are clustered based on their cosine similarities. News articles relating to the same news topic are hopefully grouped into the same cluster with a predefined similarity threshold of 0.22 . To represent the news topic, traditional TF-IDF method and P-IOLog method in Section 3 are used to weight terms to create a news-topic vector. For the TF-IDF method, we calculated the centroid vector of all news article vectors for this topic and top- $n$ words in the centroid vector which have higher TF-IDF scores were selected to create the news-topic vector, we refer to it as $\mathrm{V}_{\mathrm{TD}-\mathrm{N}}$. We also selected top- $n$ words whose term weights were calculated by the P-IOLog method of Equation 1 and the news-topic vector was created by these top- $n$ words in Equation 4, we refer to it as $\mathrm{V}_{\text {IO-N }}$. Words which are informative and tightly related to the news topic should be selected in these top- $n$ words with larger term weight than the others.

Tweets in the news-related tweet dataset are also preprocessed. Firstly, tweets which contain no hashtag or are written in non-English languages are excluded by checking the main language in the publisher's profile as "en". Secondly we filtered out all formal retweets and include tweets which have been retweeted in the dataset because Twitter users are not allowed to modify tweet contents when they use formal "Retweet" function and hashtags in those retweets may not reflect the original idea of Twitter users. At last 20,094 tweets remain after this step. Thirdly, for every hashtag in those tweets, we concatenate contents of all tweets which contain the same hashtag while mention, URL, and hashtags are removed. Hashtags appearing in less than 10 tweets are considered un-valuable and excluded. Up to now, 433 hashtags and their corresponding concatenated tweet contents have been got. We also use TreeTagger and SNER to parse concatenated contents of tweets into terms and created hashtag vectors. Here TF-IHF and P-IOLogH which have been described in Section 4 are used to weight terms and top- $n$ terms whose term weights are larger than the others are selected to create the hashtag vector. Smoothing parameter $s_{p}$ in both P-IOLog and PIOLogH are set as 0.05 . Finally we got two vectors for every hashtag: $\mathrm{V}_{\mathrm{TH}-\mathrm{N}}$ is the vector whose term weight is calculated by the TF-IHF and $\mathrm{V}_{\mathrm{IH}-\mathrm{N}}$ is the vector whose term weight is the score of P-IOLogH.

We chose "Republican", "North Korea", "Syria", and "protester" as the target words. For each target word, news topics which contain more than half of news articles including the target word are selected and summaries of these news topics are described in Table 1.

For each news topic, we set four experiments with different combinations of term weighting methods to calculate similarities between news topics and hashtags.

- Exp. 1: $\mathrm{V}_{\mathrm{TD}-\mathrm{N}} \mathrm{V}_{\mathrm{TH}-\mathrm{N}}$; term weight for news-topic vector is the TF-IDF score and term weight for hashtag vector is the TF-IHF score.

- Exp. 2: $\mathrm{V}_{\mathrm{TD}-\mathrm{N}} \bullet \mathrm{V}_{\mathrm{IH}-\mathrm{N}}$; term weight for news-topic vector is the TF-IDF score and term weight for hashtag vector is the P-IOLogH score.

- Exp. 3: $\mathrm{V}_{\mathrm{IO}-\mathrm{N}} \bullet \mathrm{V}_{\mathrm{TH}-\mathrm{N}}$; term weight for news-topic vector is the P-IOLog score and term weight for hashtag vector is the TF-IHF score.

- Exp. 4: $\mathrm{V}_{\mathrm{IO}-\mathrm{N}} \bullet \mathrm{V}_{\mathrm{IH}-\mathrm{N}}$; term weight for news-topic vector is the P-IOLog score and term weight for hashtag vector is the P-IOLogH score. 
Table 1. Summary of each news topic

\begin{tabular}{|c|c|}
\hline ID & Summary \\
\hline \multicolumn{2}{|c|}{ News topics for "Republican" } \\
\hline $\mathrm{R}_{1}$ & Iowa Republican caucus \\
\hline $\mathrm{R}_{2}$ & House Republicans refused to extend payroll tax cut bill. \\
\hline News topics for "North Korea" \\
\hline $\mathrm{NK}_{1}$ & World worried about power transition after Kim Jong-il's death. \\
\hline $\mathrm{NK}_{2}$ & World stock market affected by Kim Jong-il's death. \\
\hline $\mathrm{News}$ topic for "Syria" \\
\hline $\mathrm{S}_{1}$ & Syria allowed Arab observers into the country to end crisis. \\
\hline News topic for "protester" \\
\hline $\mathrm{P}_{1}$ & Egyptian army started to clear Tahrir Square with force. \\
\hline
\end{tabular}

In each experiment, top- $n$ words whose term weights are larger than the others for news topics and hashtags are selected to create vectors with $n$ taking the value of 50 , 100, and 200. Methods which outperform others are considered ranking those topicspecific informative words higher and hashtags recommended by these methods are considered more proper for the news topic.

\subsection{Assessments}

To evaluate recommended hashtags from four experiments, we ask assessors to judge the relevance of the recommended hashtags to each news topic. To help our assessors better understand the news topic, they could scan/search for any information if they need to make a proper decision. The whole procedure is depicted as below.

1. Three assessors are asked to read at least ten news articles which are carefully selected for each news topic so that these news articles can cover the main contents of the news topic to make them understand the news topic.

2. Top-15 hashtags with largest similarities recommended by each of four experiments are mixed to form a hashtag list for each news topic. Assessors judge the relevance of each hashtag in this list to the news topic on a three-point scale: highly relevant, partially relevant and irrelevant. They can use any tool (e.g. TagDef, Google) to find definitions for hashtags.

3. For each news topic, hashtags which are judged as highly relevant by at least two assessors are defined as highly relevant hashtags. We also define relevant hashtags as they should not be judged as irrelevant by any of assessors. Notice that highly relevant hashtags are a sub-set of relevant hashtags. 
Table 2. Recommended hashtags for $\mathrm{R}_{1}$ using top-50 words, and evaluation results

\begin{tabular}{|c|c|c|c|}
\hline Exp.1 & Exp.2 & Exp.3 & Exp.4 \\
\hline teaparty & iacaucus & teaparty & iacaucus \\
\hline teamfollowback & gop2012 & trms & trms \\
\hline tcot & ronpaul & iacaucus & gop2012 \\
\hline ronpaul & politics & ronpaul & gop \\
\hline gop & gop & gop2012 & ronpaul \\
\hline politics & trms & gop & gingrich \\
\hline trms & teaparty & tcot & politics \\
\hline iacaucus & tlot & teamfollowback & fitn \\
\hline gop2012 & fitn & gingrich & teaparty \\
\hline topnews & tcot & politics & tlot \\
\hline snn & gingrich & mittromney & huntsman \\
\hline gingrich & teamfollowback & romney & romney \\
\hline tlot & p2 & nh & fitn \\
\hline fitn & foxnews & nh & teamfollowback \\
\hline iowa & \multicolumn{2}{|c|}{ gop2012, gingrich, tcot, ronpaul, iacaucus } \\
\hline $\begin{array}{c}\text { Highly Relevant } \\
\text { Hashtags (HR) }\end{array}$ & \multicolumn{2}{|c|}{ huntsman, gop2012, gingrich, newt, tcot, politics. } \\
\hline $\begin{array}{c}\text { Relevant Hashtags } \\
\text { (R) }\end{array}$ & gop,mittromney, fitn, iowa, ronpaul, iacaucus, teaparty \\
\hline
\end{tabular}

For example, "\#iacaucus" was judged as a highly relevant hashtag because tweets containing this hashtag mainly talked about the Iowa Caucus of Republicans. However, "\#gop" which is often used to mark tweets about Republican was considered as relevant hashtags because it also relates to other Republican issues. Hashtags such as "\#topnews" used for other purposes were judged as irrelevant. Finally we got 26 highly relevant hashtags and 59 relevant hashtags for six news topics.

To evaluate performances of four experiments for six news topics, we use Precision as the evaluation metric under two-levels:

- Precision at highly relevance (P@HR): $\mathrm{P} @ \mathrm{HR}$ is the fraction of top- $\mathrm{N}_{\mathrm{hr}}$ recommended hashtags in each experiment that are highly relevant hashtags, where $\mathrm{N}_{\mathrm{hr}}$ is the number of highly relevant hashtags for the news topic.

- Precision at relevance $(\mathbf{P} @ \mathbf{R})$ : $\mathrm{P} @ \mathrm{R}$ is the fraction of top- $\mathrm{N}_{\mathrm{r}}$ recommended hashtags in the experiment that are judged as relevant hashtags, where $\mathrm{N}_{\mathrm{r}}$ is the number of relevant hashtags for the news topic.

The experiment whose P@HR and P@R values are both larger than the others should be considered as the best one for recommending hashtags. If some experiments share the same value of $\mathrm{P} @ \mathrm{HR}$, the experiment whose $\mathrm{P} @ \mathrm{R}$ value is larger outperforms. We only evaluate Precision here because Recall and F-measure share the same value in our experiments.

\subsection{Experimental Result}

We select top- $n$ words $(n=50,100,200)$ whose term weights are larger than the others by using different term weighting methods to create vectors for representing news topics and hashtags. Four experiments described in Section 5.2 are evaluated based on precision metric under two-levels for six news topics $\left(\mathrm{R}_{1}, \mathrm{R}_{2}, \mathrm{NK}_{1}, \mathrm{NK}_{2}, \mathrm{~S}_{1}, \mathrm{P}_{1}\right)$. 
Table 3. Experiment results of $\mathrm{P} @ \mathrm{HR}$ and $\mathrm{P} @ \mathrm{R}$ with their average precisions (Ave@HR, Ave@R) for six news topics $\left(\mathrm{R}_{1}, \mathrm{R}_{2}, \mathrm{NK}_{1}, \mathrm{NK}_{2}, \mathrm{~S}_{1}, \mathrm{P}_{1}\right)$ by four experiments (Exp.1 - Exp.4)

\begin{tabular}{|c|c|c|c|c|c|c|c|c|c|c|c|}
\hline & & \multicolumn{4}{|c|}{$\mathrm{P} @ \mathrm{HR}$} & \multirow[b]{2}{*}{ Ave@HR } & \multicolumn{4}{|c|}{$\mathrm{P} @ \mathrm{R}$} & \multirow[b]{2}{*}{ Ave@R } \\
\hline & & $H R$ & top50 & top 100 & top200 & & $R$ & top50 & top100 & top200 & \\
\hline \multirow{4}{*}{$\mathrm{R}_{1}$} & Exp.1 & \multirow{4}{*}{5} & 0.4 & 0.6 & 0.6 & 0.533 & \multirow{4}{*}{13} & 0.615 & 0.615 & 0.615 & 0.615 \\
\hline & Exp.2 & & 0.6 & 0.8 & 0.8 & 0.733 & & 0.692 & 0.769 & 0.692 & 0.7179 \\
\hline & Exp.3 & & 0.6 & 0.4 & 0.4 & 0.466 & & 0.692 & 0.692 & 0.615 & 0.6667 \\
\hline & Exp.4 & & 0.6 & 0.6 & 0.8 & 0.666 & & 0.769 & 0.846 & 0.692 & 0.7692 \\
\hline \multirow{4}{*}{$\mathrm{R}_{2}$} & Exp.1 & \multirow{4}{*}{2} & 1 & 1 & 1 & 1 & \multirow{4}{*}{8} & 0.625 & 0.625 & 0.625 & 0.625 \\
\hline & Exp.2 & & 1 & 1 & 1 & 1 & & 0.75 & 0.75 & 0.625 & 0.7083 \\
\hline & Exp.3 & & 1 & 1 & 1 & 1 & & 0.625 & 0.75 & 0.625 & 0.6667 \\
\hline & Exp.4 & & 1 & 1 & 1 & 1 & & 0.75 & 0.75 & 0.75 & 0.75 \\
\hline \multirow{4}{*}{$\mathrm{NK}_{1}$} & Exp.1 & \multirow{4}{*}{3} & 0.333 & 0.333 & 0.3333 & 0.333 & \multirow{4}{*}{11} & 0.454 & 0.636 & 0.636 & 0.5758 \\
\hline & Exp.2 & & 0.666 & 0.666 & 0.666 & 0.666 & & 0.636 & 0.727 & 0.727 & 0.697 \\
\hline & Exp.3 & & 0.666 & 1 & 0.666 & 0.777 & & 0.727 & 0.727 & 0.727 & 0.7273 \\
\hline & Exp.4 & & 1 & 1 & 1 & 1 & & 0.727 & 0.818 & 0.818 & 0.7879 \\
\hline \multirow{4}{*}{$\mathrm{NK}_{2}$} & Exp.1 & \multirow{4}{*}{4} & 0.5 & 0.5 & 0.25 & 0.416 & \multirow{4}{*}{8} & 0.375 & 0.375 & 0.375 & 0.375 \\
\hline & Exp.2 & & 0.5 & 0.5 & 0.5 & 0.5 & & 0.375 & 0.5 & 0.5 & 0.4583 \\
\hline & Exp.3 & & 0.25 & 0.5 & 0.75 & 0.5 & & 0.5 & 0.75 & 0.75 & 0.6667 \\
\hline & Exp.4 & & 0.75 & 0.75 & 0.75 & 0.75 & & 0.625 & 0.75 & 0.875 & 0.75 \\
\hline \multirow{4}{*}{$\mathrm{S}_{1}$} & Exp.1 & \multirow{4}{*}{4} & 0.75 & 0.75 & 0.75 & 0.75 & \multirow{4}{*}{9} & 0.666 & 0.556 & 0.556 & 0.5926 \\
\hline & Exp.2 & & 0.5 & 0.5 & 0.5 & 0.5 & & 0.666 & 0.667 & 0.556 & 0.6297 \\
\hline & Exp.3 & & 0.75 & 0.75 & 0.75 & 0.75 & & 0.666 & 0.667 & 0.778 & 0.7037 \\
\hline & Exp.4 & & 0.75 & 0.75 & 0.75 & 0.75 & & 0.777 & 0.778 & $\begin{array}{l}0.778 \\
\end{array}$ & 0.7778 \\
\hline \multirow{4}{*}{$P_{1}$} & Exp.1 & \multirow{4}{*}{8} & 0.875 & 0.75 & 0.75 & 0.791 & \multirow{4}{*}{10} & 0.7 & 0.7 & 0.7 & 0.7 \\
\hline & Exp.2 & & 0.875 & 0.875 & 0.875 & 0.875 & & 0.7 & 0.7 & 0.8 & 0.7333 \\
\hline & Exp.3 & & 0.875 & 0.875 & 0.75 & 0.833 & & 0.9 & 0.9 & 0.9 & 0.9 \\
\hline & Exp.4 & & 0.875 & 0.875 & 0.875 & 0.875 & & 0.8 & 0.9 & 0.9 & 0.8667 \\
\hline
\end{tabular}

Table 2 gives a detailed example about top-15 recommended hashtags for news topic $\mathrm{R}_{1}$ from four experiments and assessors' judgment results. For example, there are 5 hashtags considered as highly relevant hashtags by assessors $\left(\mathrm{N}_{\mathrm{hr}}\right)$. Among top-5 hashtags recommended by Exp. 4, 3 of them (iacaucus, gop2012, ronpaul) belong to the highly relevant hashtag and the precision at highly relevance (P@HR) is calculated as 3 divided by 5 . Also, there are only 2 hashtags (tcot, ronpaul) out of top-5 hashtags recommended by Exp. 1 taken as highly relevant hashtags and the P@HR should be 2 divided by 5 . Precision at relevance $(P @ R)$ is calculated in the same way. For each experiment, average precision values (Ave@HR, Ave@R) are calculated with different $n$ values. Table 3 gives out final evaluation results for four experiments among six news topics. Bold numbers are the largest average precision values for highly relevant and relevant hashtags while columns of HR and R give out the number of highly relevant and relevant hashtags for each news topic.

Exp. 4 which applies our proposed methods based on Inside and Outside assumptions to both news topics (Section 3) and hashtags (Section 4.2) has a larger average precision value than the others in most cases, which means hashtags recommended by the Exp.4 are more meaningful than hashtags recommended by other experiments. Although Exp. 4 shares the same Ave@HR value with other experiments in $\mathrm{R}_{2}$ and $\mathrm{S}_{1}$, it outperforms others in Ave@R, which also means Exp. 4 performs better. By applying our proposed methods for hashtags or news topics in Exp. 2 and Exp. 3, 
results show an improvement, although they still perform not so well compared to Exp. 4. These improvements also prove that our Probabilistic Inside-Outside Log methods for both news-topics and hashtags have positive affection to the final results. By varying the value of $n$ for top- $n$ words which are used to build the vectors, we can also observe that Exp.4 outperforms others with different $n$ values ranging from 50 to 200 , which proves that our proposed methods are more likely to rank informative words higher than the others. Applying our proposed methods only for hashtags or news topics in Exp.2 and Exp.3 could also partially improve the results with different $n$ values compared to the Exp. 1 due to the outperformance of our methods.

\section{Conclusion}

In this paper, we presented an approach for recommending hashtags in Twitter on news topics searched for an input target word. As basic components of the approach, we also proposed a method for detecting/weighting characteristic words co-occurring with the target word in news articles and two methods for detecting/weighting characteristic words co-occurring with a hashtag in tweets. Experimental results shown that our proposed methods for both news-topics and hashtags could recommend more news-topic relevant hashtags than the other methods such as TF-IDF.

The current system could recommend existed hashtags only for news topics while other topics which have been discussed in Twitter without reported by news agencies are unable to get recommended hashtags. In the future, we are planning to deal with not only news topics, but also other topics discussed by Twitter users. Also, in some cases, the current system returns too many hashtags and it is difficult for us to select appropriate hashtags from them. We think that hashtags used by influential Twitter users in the topic of interest are more likely to be recognized by others and the hashtags should be ranked higher. Finding influential Twitter users for hashtag recommendation would be another research direction we are considering.

\section{References}

1. Kwak, H., Lee, C., Park, H., Moon, S.: What is Twitter, a social network or a news media? In: Proc. of the 19th Int. Conf. on World Wide Web, Raleigh, North Carolina, USA (2010)

2. Brooks, C.H., Montanez, N.: Improved Annotation of the Blogosphere via Autotagging and Hierarchical Clustering. In: Proc. of the 15th Int. Conf. on World Wide Web, Edinburgh, UK (2006)

3. Mishne, G.: AutoTag: A Collaborative Approach to Automated Tag Assignment for Web$\log$ Posts. In: Proc. of the 15th Int. Conf. on World Wide Web, Edinburgh, UK (2006)

4. Sood, S.C., Owsley, S.H., Hammond, K.J., Birnbaum, L.: TagAssist: Automatic Tag Suggestion for Blog Posts. In: Int. Conf. on Weblogs and Social Media (2007)

5. Singhal, A., Buckley, C., Mitra, M.: Pivoted Document Length Normalization. In: 19th Annual International ACM SIGIR Conference on Research and Development in Information Retrieval (SIGIR 1996), pp. 21-29. ACM (1996)

6. Sigurbjornsson, B., van Zwol, R.: Flickr Tag Recommendation based on Collective Knowledge. In: Proc. of the 17th Int. Conf. on World Wide Web, Beijing, China (2008) 
7. Wartena, C., Brussee, R., Wibbels, M.: Using Tag Co-Occurrence for Recommendation. In: Proc. of Int. Conf. on Intelligent System Design and Application (ISDA 2009), Pisa, Italy (November 2009)

8. Belem, F., Martins, E., Pontes, T., Almeida, J., Goncalces, M.: Associative Tag Recommendation Exploiting Multiple Textual Features. In: Proc. of the 34th Int. ACM SIGIR Conf. on Research and Development in Information Retrieval, Beijing, China (July 2011)

9. Huang, J., Thornton, K.M., Efthimiadis, E.N.: Conversational Tagging in Twitter. In: Proc. of the 21st ACM Conf. on Hypertext and Hypermedia, Toronto, Ontario, Canada (2010)

10. Weng, J., Lim, E.-P., He, Q., Leung, C.W.-K.: What Do People Want in Microblogs? Measuring Interestingness of Hashtags in Twitter. In: Proc. of the 2010 IEEE Int. Conf. on Data Mining, ICDM 2010, pp. 1121-1126 (2010)

11. Correa, D., Sureka, A.: Mining Tweets for Tag Recommendation on Social Media. In: Proc. of the 3rd Int. Workshop on Search and Mining User-Generated Contents, SMUC 2011, Glasgow, Scotland, UK (2011)

12. Efron, M.: Hashtag Retrieval in a Microblogging Environment. In: Proc. of the 33rd Int. ACM SIGIR Conf. on Research and Development in Information Retrieval, ACM SIGIR 2010, Geneva, Switzerland (2010)

13. Wagner, C., Strohmaier, M.: The Wisdom in Tweetonomies: Acquiring Latent Conceptual Structures from Social Awareness Streams. In: Proc. of the 3rd International Semantic Search Workshop, p. 6. ACM (2010)

14. Zangerle, E., Gassler, W., Specht, G.: Recommending \#-Tags in Twitter. In: Proc. of the Workshop on Semantic Adaptive Social Web, UMAP 2011, Gerona, Spain (2011)

15. Phuvipadawat, S., Murata, T.: Detecting a Multi-Level Content Similarity from Microblogs Based on Community Structures and Named Entities. Journal of Emerging Technologies in Web Intelligence 3(1) (February 2011)

16. Sankaranarayanan, J., Samet, H., Heitler, B.E., Lieberman, M.D., Sperling, J.: TwitterStand: News in Tweets. In: Proc. of the 17th ACM SIGSPATIAL Int. Conf. on Advances in Geographic Information System, ACM GIS, Seattle, WA, USA (November 2009)

17. Phelan, O., McCarthy, K., Smyth, B.: Using Twitter to Recommend Real-Time Topical News. In: Proc. of the 3rd ACM Conf. on Recommender Systems, ACM RecSys, New York, NY, USA (October 2009)

18. Abel, F., Gao, Q., Houben, G.-J., Tao, K.: Semantic Enrichment of Twitter Posts for User Profile Construction on the Social Web. In: Antoniou, G., Grobelnik, M., Simperl, E., Parsia, B., Plexousakis, D., De Leenheer, P., Pan, J. (eds.) ESWC 2011, Part II. LNCS, vol. 6644, pp. 375-389. Springer, Heidelberg (2011)

19. Salton, G., Wong, A., Yang, C.S.: A Vector Space Model for Automatic Indexing. Communications of the ACM 18(11), 613-620 (1975)

20. Xiao, F., Noro, T., Tokuda, T.: Detection of Characteristic Co-Occurrence Words from News Articles on the Web. In: 21st European-Japanese Conference on Information Modelling and Knowledge Base, vol. 1, pp. 242-258 (June 2011)

21. Twitter Streaming API, https : / / dev. twitter.com/docs/streaming-api

22. Schmid, H.: Probabilistic Part-of-Speech Tagging Using Decision Trees. In: First International Conference on New Methods in Natural Language Processing, pp. 44-49 (1994)

23. Stanford Named Entity Recognizer, http://nlp.stanford.edu/software/CRF-NER.shtml 